INPLASY



To cite: Tao et al. A systematic review and meta-analysis on the efficacy and safety of immune checkpoint inhibitors combined with VEGF inhibitors in patients with solid tumors. Inplasy protocol 202130104. doi:

10.37766/inplasy2021.3.0104

Received: 28 March 2021

Published: 29 March 2021

Corresponding author:

Li Tao

taoli@ccmu.edu.cn

Author Affiliation:

Beijing Chao-Yang Hospital, Capital Medical University

Support: Beijing Chao-Yang Hospital.

Review Stage at time of this submission: Formal screening of search results against eligibility criteria.

Conflicts of interest:

None declared.

\section{A systematic review and meta- analysis on the efficacy and safety of immune checkpoint inhibitors combined with VEGF inhibitors in patients with solid tumors}

\author{
Tao, L1; Zhang, $\mathrm{H}^{2} ;$ Yao, J3.
}

Review question / Objective: P: patients with solid tumor; I: the combination therapy with immune checkpoint inhibitors and vascular endothelial growth factor inhibitors; C: other drugs or placebo; $O$ : progression-free survival or overall survival; S: RCT.

Condition being studied: The efficacy and safety of immune checkpoint inhibitors combined with VEGF inhibitors in patients with solid tumors.

Information sources: Four databases (PubMed, the Cochrane Library and Embase) were systematically searched from inception to March 2021 for randomized clinical trials (RCTs)

INPLASY registration number: This protocol was registered with the International Platform of Registered Systematic Review and Meta-Analysis Protocols (INPLASY) on 29 March 2021 and was last updated on 29 March 2021 (registration number INPLASY202130104).

\section{INTRODUCTION}

Review question / Objective: P: patients with solid tumor; I: the combination therapy with immune checkpoint inhibitors and vascular endothelial growth factor inhibitors; C: other drugs or placebo; 0 : progression-free survival or overall survival; S: RCT.

Rationale: Recently, the combination of immune-checkpoint inhibitors and antiVEGF represent the new strategy of treatment in patients with solid tumors. Our objective was to assess the efficacy and 
comparative safety of the combination of immune checkpoint inhibitor with antiVEGF for solid tumors by a systematic review and network meta-analysis.

Condition being studied: The efficacy and safety of immune checkpoint inhibitors combined with VEGF inhibitors in patients with solid tumors.

\section{METHODS}

Participant or population: Aged 20 years or older with solid tumor.

Intervention: The combination of Immunecheckpoint inhibitor (ICI) and vascular endothelial growth factor (VEGF) inhibitor.

Comparator: Other drugs (e.g: placebo or anti-VEGF alone or ICI alone or chemotherapy).

Study designs to be included: RCT.

Eligibility criteria: Inclusion: aged 18 years or older with unresectable solid tumors with adequate hematologic and end-organ function. Exclusion: History of leptomeningeal disease; Active or history of autoimmune disease or immune deficiency.

Information sources: Four databases (PubMed, the Cochrane Library and Embase) were systematically searched from inception to March 2021 for randomized clinical trials (RCTs).

Main outcome(s): All treatment-related adverse events. The progression-free survival (PFS) and overall survival of patients (OS).

Quality assessment / Risk of bias analysis: The Cochrane Risk of Bias Tool was used to assess the quality of individual studies, in accordance with the Cochrane Handbook for Systematic Reviews of Interventions. This tool considers selection bias, performance bias, attrition bias, detection bias, reporting bias, and other potential sources of bias. The overall risk of bias for each study was evaluated and rated as "low" when the risk of bias was low in all key domains; "unclear" when the risk of bias was low or unclear in all key domains; and "high" when the risk of bias was high in one or more key domains.

Strategy of data synthesis: A meta-analysis of randomized controlled trials (RCT) was performed comparing combination therapy of ICIs and anti-VEGF to other monotherapy for solid tumors to observation. The PubMed, EMBASE and Cochrane Library databases, and abstracts published in the annual proceedings were systematically searched for evidence. Relevant reports were reviewed by two reviewers independently and the references from these reports were searched for additional trials, using guidelines set by QUOROM statement criteria. Treatment-related adverse events were assessed from two different perspectives: overview and detail, based on the total number of all treatment-related adverse events and the number of each specific treatment related adverse event, respectively. Regardless of the adverse event grading, general safety was used to indicate the overview of treatment-related adverse events without distinguishing between their specific classifications. We used odds ratios and $95 \%$ credibility intervals as summary statistics to quantify the effect of dose (of $\mathrm{ICl}$ drug) or drug on the risk of grade $1-5$ and grade 3 or 4 adverse events in the network meta analysis. Odds ratios greater than one represented a safety benefit favouring the control group. Two sided $\mathrm{P}<0.05$ was considered significant. If a study reported zero adverse events in any arm, the classic half integer continuity correction (adding a 0.5 to each cell) was applied for data preparation.

Subgroup analysis: The subgroup analysis includes age, sex, geographic region.

Sensitivity analysis: 1 . Change the analysis model: when the heterogeneity is high (e.g., I squared $>50 \%$ ), the random-effects model is recommended, and the fixed-effects model is the opposite.However, my personal papers generally choose the 
random model, because the random model is relatively conservative and makes the results more safe. 2.Excluded references on a case-by-case basis: There were 8 references (1-8) for an outcome index, and the heterogeneity was changed after 1-8 were excluded on a case-by-case basis (changes in combined effect values WMD and $R R$ were also recorded). If heterogeneity changes after the first paper is found, then this paper may be the source of heterogeneity. It can be carefully analyzed why it becomes the source of heterogeneity, generally from the aspects of experimental design, sample size, outcome indicators, evaluation criteria, etc. If the heterogeneity is unchanged after 6 articles are removed, the results are relatively robust.

\section{Country(ies) involved: China.}

Keywords: Systematic review; metaanalysis; progression-free survival; overall survival; immune checkpoint inhibitors; anti-VEGF; solid tumor; adverse event.

Contributions of each author:

Author 1 - Li Tao - Preliminary searches, Piloting of the study selection process, Formal screening of search results against eligibility criteria, Data extraction Risk of bias (quality) assessment, Data analysis.

Email: taoli@ccmu.edu.cn

Author 2 - Huiyun Zhang.

Email: ashin1975126@126.com

Author 3 - Jiannan Yao.

Email: silversand1986@sina.com 